\title{
The gut-brain-axis and the heart
}

\begin{abstract}
Background: The normal physiology and pathophysiology of gut-brain, heartaxis needs further evaluation. There is evidence that brain-gut axis can influencene uropsychiatric and cardiometabolic dysfunction resulting in to obesity, insulin resistance, metabolic syndrome and cardiovascular diseases (CVDs) which is of great public health interest.
\end{abstract}

Methods: Internet search via Medline and Pub-Med and discussion with colleagues.

Results: In experimental as well as clinical studies, it has been observed, that chronic gut inflammation, predispose systemic inflammation resulting into neuropsychiatric dysfunction causing chronic anxiety disorders and memory dysfunction as well as cardiovascular dysfunction which are important risk factors of cardiometabolic diseases (CMDs). The basis of the gut-brain axis comprises; neuroanatomy and the pathophysiology represented by the vagal and spinal afferent neurons, the neuroendocrine-hypothalamic-pituitary-adrenal (HPA) axis, via the gut hormones, immune routes via the multiple cytokines, microbially-derived neurotransmitters, and finally the gate keepers of the intestinal and brain barriers. A failure in the interactions and mutual adaptations predispose a number of inflammatory, autoimmune, neurodegenerative, metabolic, mood, behavioral, cognitive, autism-spectrum, stress and pain-related disorders. Nutritional approaches; antioxidant status, microbiome manipulations, enteric and brain barrier reinforcement and sensing and trafficking modulation might improve pathophysiology of gut-brain-heart axis leading to improvement in the physical and mental health outcomes. Gut flora metabolites from foods such as choline obtained from red meat and lecithin from egg have been reported to predispose CVDs by increasing trimethyl amine $\mathrm{N}$-oxide (TAMO). Recent evidence shows that TMAO, enhances both; atherosclerosis in animal models and cardiovascular risks in clinical studies. There is a need to study the impact of targeted inhibition of the first step in TMAO generation, commensal microbial TMA production, on diet-induced atherosclerosis. A structural analog of choline, 3,3-dimethyl-1-butanol (DMB), is shown to non-lethally inhibit TMA formation from cultured microbes, to inhibit distinct microbial TMA lyases, and to both inhibit TMA production from physiologic polymicrobial cultures (e.g., intestinal contents, human feces) and reduce TMAO levels in mice fed a high-choline or L-carnitine diet. Other preventive measures early in life or corrective measures such as use of psychobiotics, fecal microbiota transplantation, and flavonoids are may be protective. A high w-6/w-3 ratio in the diet in conjunction with antioxidant flavonoids as well as probiotics in the diet in a milieu of higher BDNF levels in the gut and the brain could be important in healthy gut-brain-heart-axis for health promotion.

Conclusion: Recent evidence indicates the existence of gut-brain-axis which can interact with gut microbiota dependent on food ingestion. The gut-brain-axis can predispose systemic inflammation, more so in tissues with underlying deficiency of w-3 fatty acids and antioxidants. The pathophysiological mechanisms of gut-brainheart connection, appear to be bidirectional, resulting into neuropsychiatric diseases as well as CMDs. It is proposed that the cause of CMDs may be; existence of a gutbrain-heart-axis similar to gut-brain-axis which could be important in prevention of these diseases.

Keywords: metabolic syndrome, neural, psychological, coronary disease, hypertension, endothelial dysfunction, atherothrombosis, inflammation
Volume 7 Issue 3 - 2018

Ram B Singh,' Jan Fedacko, ${ }^{2}$ Dominik Pella, ${ }^{3}$ Daniel Pella, ${ }^{2}$ Peter Jarcuska, ${ }^{2}$ Viliam Mojto, ${ }^{4}$ Al-bawareed OA, ${ }^{5}$ Sergey Chibisov, ${ }^{6}$ Elena $V$ Kharlitskaya, ${ }^{7}$ Maria Abramova ${ }^{8}$

'Halberg Hospital and Research, India

${ }^{2}$ Faculty of Medicine, PJ Safaric University, Slovakia

${ }^{3}$ Eastern Slovak Institute of Cardiovascular Sciences, Slovakia

${ }^{4}$ Faculty of Medicine, Comenius University, Slovakia

${ }^{5}$ Department of Normal Physiology, People's, Friendship University of Russia, Russia

${ }^{6}$ Department of Pathophysiology, People's Friendship University of Russia, Russia

${ }^{7}$ Veterinary Medicine of the Agrarian and Technology Institute, Peoples, Friendship University of Russia, Russia

${ }^{8}$ Department of Anatomy, People's Friendship University of Russia, Russia

Correspondence: Ram B Singh, Halberg Hospital and Research Institute, Civil Lines, Moradabad (UP) 24400 I, India, Tel +0091-59I-24I7437,Email rbs@tsimtsoum.net

Received: May 15, 2018 | Published: June 14, 2018

\section{Introduction}

In a landmark experiment in 2008, Wang et al., ${ }^{1}$ demonstrated that direct administration of lipids into the upper intestine, enhances the concentration of upper intestinal long-chain fatty acyl-coenzyme A (LCFA-CoA) and suppressed glucose production by activation of a gut-brain-liver neural axis to regulate glucose homeostasis. Previous studies also suggested that upper gut lipids inhibit food consumption by the activation of an gut-brain axis and it is possible that a brain- liver axis may find out blood lipoproteins for inhibition of the production of glucose..$^{2-4}$ These experiments indicate the existence of a gut-brain-liver neural axis which may influence patho-physiology of cardiovascular and metabolic function. ${ }^{5-8}$ The effects of gut microbiota on behavior are evident, because of the involvement of serotonin and tryptophan neurotransmitters, indicating a possibility that changes in the intestine could be may be essential in the physiology pathology of neuropsychiatric problems..$^{5-8}$ The gut-brain neural axis is a bidirectional system connecting the brain and gut, linking emotional 
and cognitive centers of the brain to modulate targetted functioning of the gastro-intestinal tract and cardiovascular system. Therefore, disturbances in gut microbiota can have adverse effects on gut-brainaxis as well as on gut metabolites resulting in to increased risk of cardiovascular events. ${ }^{9-12}$ Similarly, chronic anxiety disorders are known to predispose cardiovascular diseases [CVDs) which can also be bidirectional. ${ }^{13-17}$ Chronic and acute emotional stress can also damage certain areas of the brain that are known to predispose inflammation in the arteries, leading to atherosclerosis. ${ }^{13}$ Therefore, many experts believe that atherosclerosis may be considered, as if it is a disease of the brain (Table 1), (Figure 1), (Figure 2). Al and mark cohort study has confirmed that increase in amygdalar activity was significantly associated with greater bone-marrow activity, arterial inflammation, increase in C-reactive protein leading to increased risk of CVD events. ${ }^{18}$ A previous study showed that it is lateral amygdala which may be responsible for inflammation in the arteries..$^{19}$ Recently gut microbiota induced metabolites have been reported to cause systemic inflammation resulting in to heart attack and heart failure. ${ }^{20}$

Table I Pathways of communication between gut microbiota, brain and the heart

I. The gut-brain's neural network,

2. Gut immune system,

3. Neuroendocrine-hypothalamic-pituitary-adrenal axis,

4. Some neurotransmitters and neural regulators synthesized by gut bacteria, and

5. Barrier paths including intestinal mucosal barrier and bloodbrain barrier.

6. Effects of gut flora metabolite on body systems.

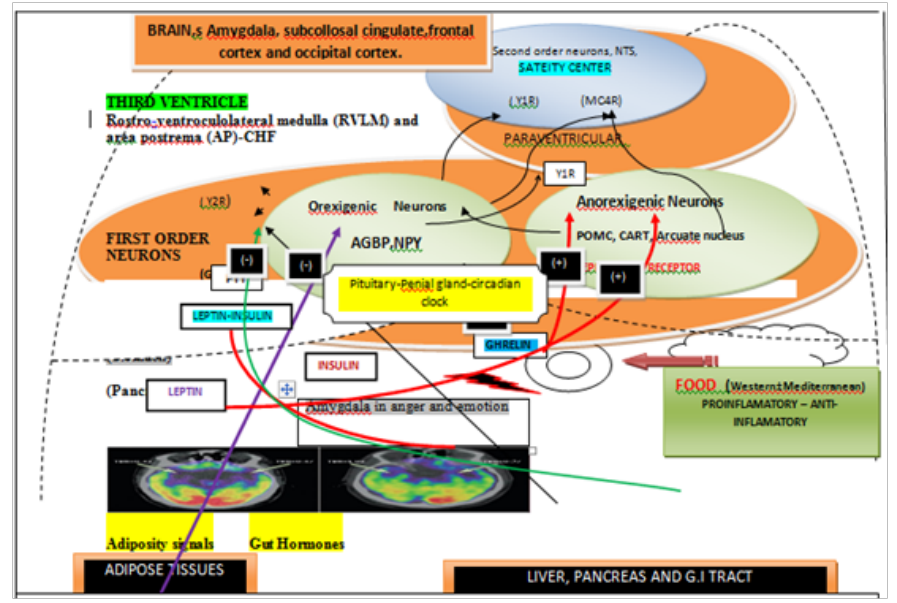

Figure I Gut-brain-heart interactions in controlling of feeding behavior in human.

Hormonal circuits from the gut involving stomach, small intestine, and pancreas and Adipose Tissue influence the sensations of hunger and satiety exerted via hypothalamic neuroendocrine pathways; POMC, Arcuate nucleus. GHSR (growth hormone secretagogue receptor to which ghrelin binds). MC3R and MC4R melanocortin 3 receptor and melanocortin 4 receptor). YIR (neuropeptide Y (NPY) receptors I) and Y2R (NPY receptors 2). Ghrelin from the stomach, leptin from adipose tissue, insulin from the pancreas, and peptide tyrosine tyrosine (PYY) from the small intestine bind to receptors on orexigenic and/or anorexigenic neurons in the ARC of the hypothalamus.

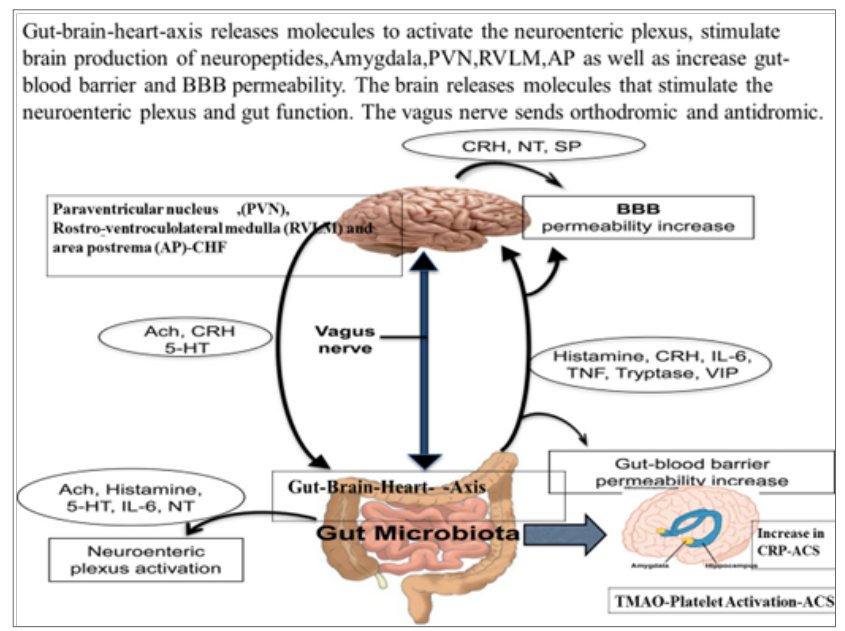

Figure 2 Gut microbiota-brain-axis release molecules that can activate the neuroenteric plexus, stimulate brain production of neuropeptides, POMC,AN and amygdala resulting in to heart disease.

\section{Objectives}

This review aims to examine whether gut-brain-axis can influence pathophysiology of cardiovascular function resulting in to CMDs and whether cardiometabolic dysfunction can have adverse effects on neuropsychiatric function and related diseases.

\section{The gut-brain-axis}

The brain can influence gastrointestinal system via central circadian clock and other areas of the brain. As mentioned above, the gut-brain axis is an interface for both directions between the brain and gut, linking mental and cognitive centers of the brain with the functioning of the gastrointestinal tract and other body systems. ${ }^{5}$ The alterations in the gut may be essential in the physiopathology of neuropsychiatric disorders due to involvement of tryptophan and serotonin release by gut microbiota. The mood and cognition are under influence of the serotonergic system which is a diffuse network within the central nervous system. The release of tryptophan levels can be altered by depletion or supplementation of certain agents, used for modification of serotonin levels responsible for mood alteration. The role of serotoninin higher order brain function, particularly in relation to food intake have allowed us to conclude that deficiency in brain serotonin levels may predispose poor memory and depressed mood responsible for depression and memory dysfunction. ${ }^{5,8}$ Figure 1 shows the hormonal circuits from the gut involving stomach, small gut, pancreas and adipocyte may influence the sensations of hunger and satiety exerted via hypothalamic neuroendocrine pathways. The precise mechanisms on above interactions and connections have not yet been fully clear but it has been reported that gut microbiota affects the brain's physiological, behavioral, and cognitive functions. ${ }^{8}$ It is possible that gut-brain axis includes microbiota in the intestines as well as products of metabolism in conjunction with gut nervous circuit, branches of sympathetic and parasympathetic nerves as component of the autonomic nervous system. Other important parts could be neuroendocrine and neural-immune system as well as central nervous system. The functional characteristics of gut microbiota may be due to its composition which may be regarded as microbiome. The gut microbiota can be further characterized by metagenomics which is 
an special technique for study of genetic aspects of organisms present in the gut. Recently, gut flora metabolite has been found to influence cardiovascular function., ${ }^{9} 10$ The gut microbiota and the brain may have five routes for communication which appear to be bidirectional as given in the Table 1 .

\section{The gut-brain-heart axis}

There is a complex nutrient sensing mechanisms and an integration of neuronal and hormonal signaling via gut-brain axis and the heart. ${ }^{5,9,10,21-31}$ The gut and brain closely communicate to modulate energy homeostasis and metabolism in response to food intake. ${ }^{22}$ There are distinct mechanisms in the brain and gut for sensing lipid availability, which triggers subsequent regulation of feeding, glucose homeostasis, and adipose tissue metabolism. Recent studies indicate that parasympathetic and sympathetic signaling, influences the central receptors, neuropeptides, and gut hormones that communicate with the brain, have been shown to modulate hepatic and intestinal lipoprotein metabolism. ${ }^{22}$ The glucagon-like peptides obtained from the gut appear to be particularly important in modulating the release of chylomicron particles from the gut, via a novel brain-gut axis. It is clear that the central and enteric nervous systems can influence several aspects of lipid and lipoprotein metabolism due to a bidirectional communication between the gut and the brain. Dysregulation of these pathways may predispose postprandial dyslipidemia and insulin resistance resulting in to increased risk of cardiometabolic diseases (CMDs).

It is known that in diabetic dyslipidemia with insulin-resistance, overproduction of atherogenic chylomicron particlespost-prandially in the gut is important which indicate that in addition to liver, gut dysfunction also influence cardiovascular dysfunction. ${ }^{21-24}$ Apart from increase in the release of insulin, stimulation of peripheral receptor of glucagon-like peptide-1 (GLP-1) may provide additional benefit of reducing overproduction of chylomicron among subjects suffering from diabetes mellitus. ${ }^{21}$ The presence of receptors and neurones in the central nervous system that increase GLP-1, poses the possibility, that central GLP-1 may influence the production of these potentially atherogenic particles. In an experiment in syrianhamsers, a single intra-cerebro-ventricular injection of the GLP-1 receptor agonist exendin-4 was associated with reduction in lipoprotein-triglyceride which is rich in triglyceride and in accumulation of -apolipoprotein B48, compared to controls. It is possible that central GLP-1 is a novel regulator of chylomicron production via melanocortin-4 receptors. In the development of diabetic dyslipidemia and chylomicron overproduction, there may be a relative importance of central accessibility of therapies based on GLP-1, with important role of brain-gut axis. ${ }^{21}$ These therapies could be protective against CMDs including CVDs which may influence gut functions. ${ }^{24-29}$

The gut has been called the mirror of mind-brain function as well as dysfunction in most of the disciplines of internal medicine. An experimental study in mice indicated that gastrointestinal inflammation can induce anxiety-like behaviour and altered biomarkers related to the brain dysfunction that may be due to inflammation in the brain. ${ }^{30,31}$ There is evidence from neuro-immunology that treating viral hepatitis with interferon, could trigger severe depression which indicated that circulating pro-inflammatory cytokines can play a significant role in mood and mood disorders. ${ }^{32}$ This relationship may also contribute to the high prevalence of depression in patients with inflammatory bowel disease as well as among patients with cardiovascular diseases and type 2 diabetes mellitus..$^{33}$ Table 2 shows the brain areas responsible for concerned psychological behavior. The association of inflammation with metabolic syndrome may be the predictor of cardiovascular diseases (CVDs) and type 2 diabetes.

Table 2 Effects on specific neuro-anatomical region activation via concerned emotions

\begin{tabular}{cll}
\hline No. & Neuronal region & Mental dysfunction \\
\hline I. & Medial frontal cortex & Emotional processing \\
2. & Amygdala & Fear and anger \\
3. & Subcallosal cingulate & Sadness \\
4. & Occipital cortex and amygdala & $\begin{array}{l}\text { Emotional induction by } \\
\text { visual stimuli }\end{array}$ \\
5. & Anterior cingulate and insula & $\begin{array}{l}\text { Induction by emotional } \\
\text { recall imagery }\end{array}$ \\
6. & Anterior cingulate and insula & $\begin{array}{l}\text { Emotional task with } \\
\text { cognitive demand }\end{array}$ \\
\hline
\end{tabular}

In experimental studies, animals did not display signs of general malaise, but still exhibited patterns that are a rodent correlates of anxiety and treatments ranging from probiotics to anti-tumor necrosis factor (TNF) antibodies, differentially altered inflammation, and behavior. ${ }^{31-34}$ Blocking of TNF signaling blunted inflammation and decreased the level of circulating cytokines leading to normalized behavior. ${ }^{31,32}$ The steroid budesonide was not quite as effective, but showed a similar direction of change(s). The probiotics decreased the markers of gut inflammation, but did not affect circulating cytokine levels. However, bifidobacteria was about as effective as the immunesuppressants in improving behavior which was associated with changed expression of an important neurotrophic factor in a brain region that is responsible for learning and memory. ${ }^{35}$ In contrast, a murine strain of lactobacillus was ineffective. It seems that the braingut axis is bidirectional and includes more than just the sensory input that certainly influences mood and behavior. ${ }^{36}$ It may be possible to target the neuroimmune connection by influencing intestinal inflammation, permeability, gut contents and/or the microbial flora and food consumption. ${ }^{31-38}$ Increased intestinal permeability has been associated with subtle changes in cytokine levels as well as chronic fatigue and fibromyalgia, ${ }^{34}$ which was selective for one of the two organisms tested, and this was similar to results obtained in humans. ${ }^{38}$ However, the benefit was independent of the changes in the circulating cytokines, indicating that other factors related to neural mechanisms may be important. There may be decline in blood glucose production, due to activation of N-methyl-D-aspartate (NMDA) receptors in the dorsal vagal complex which may also neutralize fast food induced hyperglycemia. ${ }^{39}$

\section{Nutritional aspects of gut-brain-axis}

The intake of food according to time structure may influence central circadian clock as well as peripheral clocks in the gut which have been demonstrated to alter energy metabolism and cardiovascular function. ${ }^{36,37}$ Increased intake of energy in the morning is associated with weight loss whereas eating same amount of food in the evening caylused gain in weight possibly because of variations in basal metabolic rate and glucocorticoids. Long chain 
polyunsaturated fatty acids (PUFA) such as w-3 fatty acids are known to inhibit neurodegeneration and pro-inflammatory cytokine induced inflammation. ${ }^{39,40}$ Leptin and cholecystokinin may also have interactions with incretins and may be inversely associated with long chain polyunsaturated fatty acid (PUFA) in the diet and in the tissues. Omega-3 fatty acid as well as antioxidant flavonoid/ polyphenols deficiency of the brain can independently have adverse effects on myocardium and vascular cells, in the form of a trigger that lies in the brain, and produce a chronic electrical instability in arterial cells causing coronary artery spasm and in the myocardium causing ventricular arrhythmias. ${ }^{41-43}$ Lipoprotein associated phospholipase $\mathrm{A} 2^{44}$ is a new proinflammatory marker under the influence of proinflammatory diets characterized with high w-6/w-3 ratio, trans fat and high saturated fat and refined carbohydrates..$^{45-50}$ Recent studies indicate that $\mathrm{w}-3$ fatty acids in the tissues were inversely associated with CAD ${ }^{51-53}$ Further studies showed that Mediterranean diets rich in w-3 fatty acids and antioxidant flavonoids were protective against cardiac events and metabolic syndrome which can be explained in part due to beneficial effects dietary fiber, antioxidants and w-3 fatty acids of the diet on gut microbiota, neurones and the cardiovascular tissues. ${ }^{54-61}$ However, only a few studies have examined the role of brain related mechanisms in the protection provided by Mediterranean diets. It is quite possible that these diets act via gut-liver-brain and heart connection, at least partially in providing such benefits. It appears that the gut may act as a neuroendocrine organ that responds to food intake, and large meals rich in trans fat, w- 6 fat and refined carbohydrates can cause inflammation and have adverse effects on the brain-gut and heart connection. Gut also releases several hormones; ghrelin, leptin, cholecystokinin and brain-derived neurotrophic factor which can influence neuronal growth factor and brain function.

Recently, a human gut microbial gene catalogue was also found in the body, which appears to be established by metagenomics sequencing. The human genome has 23,000 genes but the human microbiome has more than 1.00 million genes. However, the gut microbiota may be comprised of the trillions of microbes which inhabit the gut and may be considered a complex bioreactor with several metabolic and immunological effects. ${ }^{10,20}$ These beneficial effects extend beyond the gut itself, and, in the last decade, an increasing amount of evidence has linked functions and alterations of the gut microbiota to brain diseases as well as CMDs. ${ }^{6-10}$ The host metabolic mechanisms are under strong influence of interactions between dietary factors and gut microbes Figure 2 shows the gut microbiota-brain-axis releasing molecules that stimulate production of neuropeptides in brain, can activate the neuroenteric plexus, POMC,AN, circadian clock and amygdala resulting in to heart disease. The increased absorption of energy from the gut content may contribute to obesity, a trait that is transmissible in animal models, suggesting a vital role for the gut microbiome which is partly mediated by short chain fatty acids (SCFAs).$^{6-8}$ Gut metabolites from foods such as choline obtained from red meat and lecithin from egg have been reported to predispose cardiovascular diseases by increasing trimethyl amine N-oxide (TAMO). ${ }^{10-13}$

\section{TMAO and cardio-metabolic diseases}

TMAO, a gut-microbiota-dependent metabolite, enhances both; atherosclerosis in animal models and cardiovascular risks in clinical studies. ${ }^{10-12}$ There is a need to study the impact of targeted inhibition of the first step in TMAO generation, commensal microbial TMA production, on diet-induced atherosclerosis. A structural analog of choline, 3,3-dimethyl-1-butanol (DMB), is shown to non-lethally inhibit TMA formation from cultured microbes, to inhibit distinct microbial TMA lyases, and to both inhibit TMA production from physiologic polymicrobial cultures (e.g., intestinal contents, human feces) and reduce TMAO levels in mice fed a high-choline or L-carnitine diet. ${ }^{12}$ Further evidence showed that DMB inhibited choline diet-enhanced endogenous macrophage foam cell formation and atherosclerotic lesion development without alterations in circulating cholesterol levels. ${ }^{12}$ Therefore, targeting gut microbial production of TMA specifically and non-lethal microbial inhibitors in general may serve as a potential therapeutic approach for the treatment of CMDs. Figure 3 shows the use of dimethyl butanol (DMB) to inhibit gut microbial trimethylamine production for the treatment of atherosclerosis. ${ }^{3}$

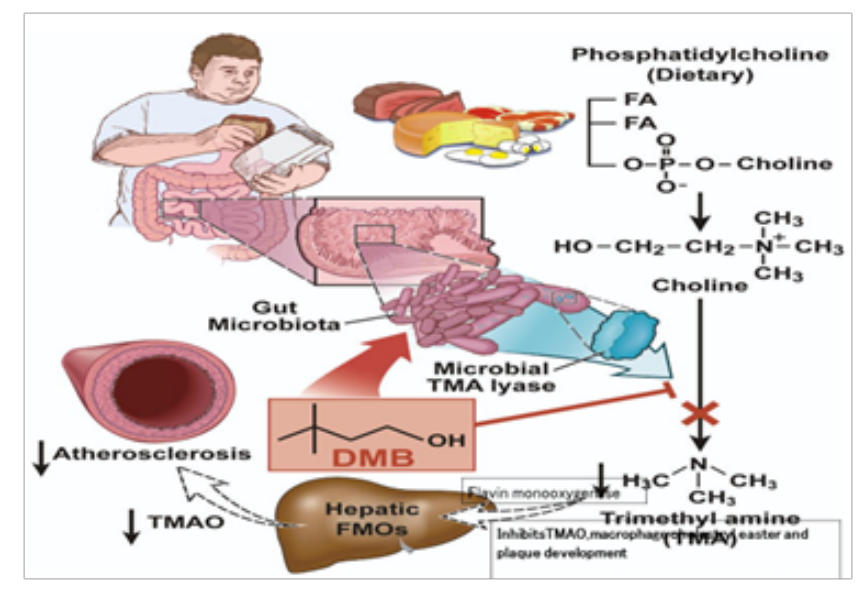

Figure 3 Use of dimethyl butanol (DMB) to inhibit gut microbial trimethylamine production for the treatment of atherosclerosis. ${ }^{3}$

The development of phenotypes in the host among humans could manipulated by the gut microbiome community that is complex and metabolically active. ${ }^{62-72}$ The Metabolic Syndrome In Men (METSIM) study involving 531 Finnish men revealed consistent relation between fasting serum levels of a number of metabolites; fatty acids, amino acids, lipids, and glucose with gut microbiota. ${ }^{72}$ There were significant associations of coronary artery disease (CAD) and stroke with fasting plasma TMAO levels. It is possible that the content of the gut microbiota and their metabolites in the blood could be biomarkers for future research to understand the relationship of host and gut microbiota. It is known that TMAO, a metabolite derived from gut microbes and dietary phosphatidyl- choline and L-carnitine, may be associated with pathogenesis of CAD and risk of CVDs. ${ }^{73}$ There was no study to demonstrate that plasma TMAO can predict mortality risk in patients with stable CAD. The relationship between fasting plasma TMAO and all-cause mortality was examined among 2235 patients with stable CAD during a follow up of 5 -year. ${ }^{73}$ The results revealed that increased concentration of TMAO were associated with an increased risk of death, after adjustments for other risk factors such as estimated glomerular filtration rate and high-sensitivity C-reactive protein. The concentration of TMAO was the main indicator of incident risk of deaths following cardio-renal and inflammatory biomarker adjustments and provided significant incremental prognostic value for deaths due to all other causes. It is possible that higher plasma concentration of TMAO can predict higher long-term risk of death among patients with stable CAD treated with optimal drug therapy. ${ }^{73}$ 
TMAO, a gut microbial-dependent metabolite may be also elevated in chronic kidney diseases (CKD), apart from CVDs and may be associated with pathogenesis of CAD in patients with. ${ }^{74-76}$ The relationship between fasting plasma TMAO and all-cause mortality over 5-year follow-up in 521 stable subjects with CKD (estimated glomerular filtration rate [eGFR] $<60 \mathrm{ml} / \mathrm{min} / 1.73 \mathrm{~m}^{2}$ ) was examined. Median TMAO level among CKD subjects was $7.9 \mu \mathrm{M}$ (interquartile range $5.2-12.4 \mu \mathrm{M})$, which was markedly higher $(\mathrm{P}<0.001)$ than in non-CKD subjects $(n=3,166)(74)$. A higher concentration of TMAO level was associated with a 2.8 -fold increased mortality risk, after adjustments for traditional risk factors, hsCRP and eGFR, elevated TMAO levels remained predictive of 5-year mortality risk (HR 1.93 [95\%CI 1.13-3.29], $\mathrm{p}<0.05$ ). TMAO provided significant incremental prognostic value. It seems that plasma TMAO levels are both elevated in patients with CKD and portend poorer long-term survival and increased dietary intake of foods that increase TMAO appear to directly contribute to progressive renal fibrosis and dysfunction (74). Microflora-dependent trimethylamine-N-oxide (TMAO) formation, which results from intake of choline and L-carnitine-rich food, shows promise as a predictor of CVD risk, but these associations have not been examined in ethnically diverse populations. In a multiethnic population-based study of adults involving 1285 adults, the stability of TMAO and L-carnitine in stored serum samples and their association with intimal medial thickness, prevalent risk factors, and clinical events were examined. ${ }^{75}$ In 292 consecutive individuals (99 CVD cases and 193 unmatched control subjects), L-carnitine and TMAO concentrations were assessed.The mean $( \pm \mathrm{SD})$ TMAO level was $1.998 \pm 3.13 \mu \mathrm{M}$ and L-carnitine was $42.29 \pm 11.35 \mu \mathrm{M}$. TMAO levels showed a significant, graded association with prevalent CVD (odds ratio, 3.17; 95\% confidence interval, 1.05-9.51; $P$ trend $=0.02$ ), whereas no significant association of 1 carnitine was observed. The findings revealed an association between TMAO with prevalent CVD in a multiethnic population.

\section{TMAO levels and risk of acute coronary syndromes}

The prognostic value of the gut-derived metabolite, TMAO was confirmed for the first time, using 1-year major adverse cardiovascular events (MACE) risk, in a large Swiss cohort of ACS undergoing coronary angiograph. ${ }^{10}$ High plasma levels of TMAO in patients with ACS can independently predict major adverse cardiac events (MACE) at 30 days and 6 months and mortality at 7 years, also in United States. ${ }^{10}$ In two independent cohorts of ACS, the relationship of TMAO levels with incident cardiovascular risks among sequential patients was examined. ${ }^{10}$ The Cleveland Cohort, comprised of 530 patients with suspicion of cardiac pain in the chest and high plasma levels of TMAO at clinical presentation was significant risk factor for MACE such as myocardial infarction, stroke, need for revascularization, or death during the 30 -day follow up $(\mathrm{P}<0.01)$ and 6 -month $(\mathrm{P}<0.01)$ intervals. The concentrations of TMAO were also a significant $(\mathrm{P}<0.05)$ predictor of the 7-year mortality. In subjects with lower troponin T, the levels of TMAO at baseline presentation also showed increased risk of incident MACE over the 30 days and 6 months, $(p<0.01)$ of follow up. An independent multi-center Swiss Cohort of ACS $(n=1683)$ who underwent coronary angiography, assessed the prognostic value of TMAO. The levels of TMAO predicted an enhanced risk of MACE at 1-year, of follow up $(\mathrm{P}<0.05)$. It is clear that plasma levels of TMAO among patients with chest pain can predict both near- and long-term risks of incident cardiovascular events. TMAO levels may provide clinical utility in risk stratification among subjects presenting with suspected ACS

In another study among patients with heart attack, concentrations of TMAO were independently associated with death or myocardial infarction at 2 years after hospitalization. ${ }^{11}$ Suzuki and his colleagues examined TMAO levels in plasma among 1079 patients of AMI admitted to the University Hospitals of Leicester between August 2004 and April 2007. Multivariable analyses revealed TMAO was an independent predictor of death/MI at 2 years (HR 1.21, 95\% CI $1.03-1.43 ; P=0.023)$ but not at 6 months $(P=0.119)$. Plasma levels of TMAO was the only biomarker that significantly predicted outcome, despite inclusion of such contemporary markers as cardiac troponin, $\mathrm{N}$-terminal probrain natriuretic peptide (NT-proBNP), and copeptin, during the follow up of two years. The Global Registry of Acute Coronary Events (GRACE) score for calculating death/MI at 6 months, also revealed that plasma TMAO was able to down-classify patients with low risk (NRI 29.0, 95\% CI 19.8-38.3; $P<0.0005$ ) but unable to reclassify those at high risk (NRI-9.1, 95\% CI -30.0 to 11.8 ). These results are quite interesting and provide evidence base for TMAO beyond stable patients, including those with heart failure or peripheral artery disease, and indicate new prospect of TMAO as a potentially modifiable risk marker for cardiovascular events.

These findings also indicate new perspectives in terms of intervention that need additional research, to find out their influence on TMAO levels directly either by dietary intervention. It is possible that in the near future, new compounds inhibiting the enzyme in the bacteria that produces TMAO, may be used for therapy for prevention of ACS and CMDs. ${ }^{11}$ It may be proposed that some of the dietary components; omega-3 fatty acids, resveratrol, curcurmin, dietary fiber, probiotics etc that are known to influence CVDs, may be examined for their beneficial effects on TMAO and other enzymes. TMAO is produced after gut bacteria break down dietary nutrients such as phosphatidylcholine, choline, and L-carnitine, found in red meat, eggs, and high-fat dairy products, all common in the Western diet. Hence nutrients rich in Mediterranean style diets may be considered for future therapy because such diets have been proven to provide beneficial effects in patients after ACS. It has also been already demonstrated that targeting trimethylamine (TMA), the first step in TMAO formation, with 3,3-dimethyl-1-butanol inhibits foamcell formation and aortic atherosclerotic plaque formation in mice, without altering normal gut flora. ${ }^{12}$

In United States cohort, patients with the highest TMAO levels faced a nearly twofold increased hazard of 7-year mortality when compared with those with the lowest level (OR 1.81, 95\% CI $1.04-3.15 ; P<0.05)(10)$. It is important that in adjusted analyses of patients with initially negative cardiac troponin, elevated TMAO levels maintained prognostic significance for MACE at 30 days (OR 5.83, 95\% CI 1.79-19.03; $P<0.01$ ) and 6 months (OR 5.51, 95\% CI $1.90-16.01 ; P<0.001)$ beyond traditional risk factors, biomarkers, and ECG data, including C-reactive protein (CRP), hypertension, hyperlipidemia, and diagnosis of STEMI, non-STEMI, or unstable angina. It is possible that the ability to generate rapid and accurate TMAO results through point-of-care testing could significantly improve rapid triaging and risk stratification among subjects presenting with suspected ACS. It has been observed that there may be intraindividual variation in TMAO, spiking, for example, in patients after simply eating a steak dinner. ${ }^{11}$ However, some of the variations in TMAO concentrations, similar to CRP, may be attributed to circadian 
dysfunction due to late night sleep and late night eating which are known to alter circadian metabolism in liver and pancreas resulting in to CMDs. In view of the variability in TMAO levels, it may not be so useful as a diagnostic marker but as a prognostic marker.

The Swiss diet among Swiss patients appear to be quite a different diet, and this is of interest because TMAO levels are influenced by diet, in particular meat, shellfish, and eggs. Since American diets are quite rich in these foods, TMAO levels were much higher in patients in the US in association with higher cardiovascular complications in these patients, showing a graded increase in incident MACE risk. ${ }^{10}$ The adjusted risk of MACE at 1 year was 1.5 times higher in patients with the highest TMAO levels $(>4.85 \mu \mathrm{M})$ than in those with the lowest levels of TMAO $(0.08-1.93 \mu \mathrm{M})$ (hazard ratio [HR] 1.57, 95\% CI 1.03-2.41; $P<0.05)$. Inclusion of TMAO levels in the Cleveland Clinic's model significantly improved risk estimation for MACE at 30 days and 6 months, with significant improvement in $C$ statistics for both time points which provides a further proof that TMAO levels may be a risk factor of ACS. ${ }^{10}$ Further evidence regarding role of gutbrain-heart-axis comes from a cohort study showing that increase in amygdalar activity was significantly associated with greater bonemarrow activity, arterial inflammation, increase in C-reactive protein and increased risk of CVD events. ${ }^{18}$ In a cohort study among 293 subjects, aged 30 years or older, without any history of heart and vascular disease, activities of amygdala, bone-marrow and arterial inflammation were examined by functional magnetic resonance imaging(fMRI). After a median follow-up of 3.7 years, the results showed that increase in the activity of amygdala in the form of tremors, was significantly associated with greater activity in the bonemarrow $(r=0.47, p<0.0001)$, increase in arterial inflammation $(r=0.49$, $\mathrm{p}<0.0001$ ), with an increased risk of CVD events (standardized hazard ratio: $1.59,95 \% \mathrm{CI}: 1.27-1.98, \mathrm{p}<0.0001$ ). Figure 4 shows the mental dysfunction-induced increased amygdaler activity in humans leading to systemic inflammation (modified from Tawakol et al. ${ }^{8}$ It is possible that amygdalar activity can worsen and cause increased cardiovascular damage in presence of antioxidant deficiency in the neurones, gut and the heart tissues.

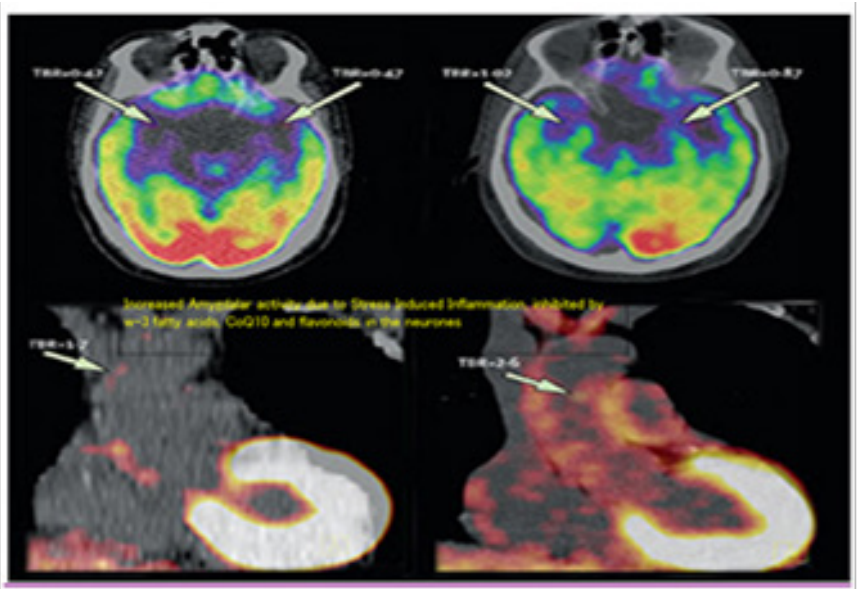

Figure 4 Mental dysfunction-induced increased amygdaler activity in humans leading to systemic inflammation. Tawakol et al. ${ }^{18}$

\section{Mechanisms}

The formation of TMAO in our body takes place following nutrient ingestion, via gut microbes which form TMA, and then host hepatic flavin monooxygenases (FMOs) catalyze the conversion of TMA into TMAO. ${ }^{12}$ Recent studies highlight the importance of both TMAO and host hepatic FMO3, the primary FMO responsible for TMAO production, as important regulators in host lipid and sterol metabolism, as well as in development of atherosclerosis ${ }^{12,76}$ Figure 1. A structural analogue of choline, 3,3-demethyl-1-butanol (DMB) is an inhibitor of microbial TMA production (a choline TMA lyase inhibitor). It also inhibits choline diet dependent enhancement in TMAO, endogenous macrophage foam cell formation, and in development of atherosclerosis.

\section{Fatty acids in the diet and gut dysfunction}

SCFAs are end-products of microbial fermentation of dietary fibers, and involved in energy harvest from the gut as well as maintaining the integrity of the gut mucosa. ${ }^{20}$ Decline in certain SCFAs, in particular butyrate, may result in a gut dysfunction; mucosal barrier, facilitating passive leakage of microbial toxins such as lipopolysaccharides (LPSs) and chylomacrons from the gut to surrounding adipose tissue and circulation, triggering inflammation and insulin resistance. ${ }^{25}$ In animals, increased production of hepatic apoB100-containing VLDL particles has been demonstrated to cause dyslipidemia and in humans with insulin resistance leading to metabolic syndrome and type 2 diabetes, and contributes to the typical dyslipidemia of these conditions. ${ }^{30}$ In addition, postprandial hyperlipidemia and elevated plasma concentrations of intestinal apoB48-containing chylomicron and chylomicron remnant particles have been demonstrated in insulin resistant which is a risk factor of CMDs. The intestinal production of lipoprotein could be primarily due to the ingestion of fat and its absorption. However, recent studies indicate a significant role of the intestine itself in regulating the production of triglyceride-rich lipoproteins (TRL) which are further dysregulated due to insulin resistance. ${ }^{21-25,30}$

Recent studies indicate that there is greater production of intestinal lipoproteins that are rich in apoB48 which can regulate hepatic lipoprotein particle production. ${ }^{25,30}$ Further it has been recently observed, that release of intestinal lipoproteins are regulated by monosacharides, plasma free fatty acids (FFA), antioxidant resveratrol, intestinal peptides (e.g. GLP-1 and GLP-2), and pancreatic hormones (e.g. insulin). ${ }^{30}$ Recent studies support the hypothesis that the small intestine is an absorptive organ as well as it plays an active role in regulating the rate of production of chylomicrons in fed and fasting states which can inhibit beta cell function and influence cardiovascular function. In insulin resistance and type 2 diabetes, metabolic signals and in some cases an aberrant intestinal response to these factors may be additive to the increased synthesis and release of TRL. It is clear from the recent evidence that production of intestinal lipoprotein is a process with metabolic plasticity and that modulation of intestinal lipoprotein release may be a feasible therapeutic strategy in the treatment of dyslipidemia and possibly prevention of athero-thrombosis and CMDs. Regulation of intestinal production of lipoprotein appears to be important for the development of new strategies for the management of dyslipidemia. ${ }^{30}$

The bidirectional communication between the central nervous system and cardiometabolic system with gutmicrobiota, may be considered as the gut-brain-heart-axis., , $3,5,9-13,75$ Further evidence indicate that gut microbiota may be linked to a triple burden of diseases; neuropsychiatric diseases, gastrointestinal diseases as well as CMDs. Alterations in gut microbiota and inflammation of the gut have been linked to predispose several mental disorders; such as aggression, 
anxiety and depression as well as cognitive deficit and dementia. ${ }^{5-8}$ It is possible that ingestion of probiotics may restore normal microbial balance, and may have a protective role in the management of diseases of gut, brain and the heart. The development of the gut microbiota via probiotics and prebiotics or simply by a Mediterranean style diet may be linked to decline in dysbiosis, leading to reduction in mental disorders such as anxiety and depression and CMDs. A healthy gut function has been linked to normal central nervous system (CNS) function, gut hormones, neurotransmitters and immunological factors released from the gut are known to send signals to the brain either directly or via autonomic neurons. ${ }^{5-8}$ Sudo and co-workers demonstrated the existence of the gut-brain axis in the landmark study that discovered the impaired stress response in germ-free mice. Other studies using germ-free mice also supported this existence, as well as the idea that the gut-brain-axis (GBA) extends even beyond these two systems into the endocrine, neural, and immune pathways..$^{1-4,77-80}$ Now Singh and coworkers propose the possibility of the existence of gutbrain-heart-axis due to new findings showing that gut flora metabolite can predispose CMDs. ${ }^{2,3,9,13}$

The emergence of a microbiota-gut-brain axis to describe the complex networks and relationship between the gastrointestinal microbiota and host reflects the major influence this environment may have in brain health and disorders of the central nervous system $(\mathrm{CNS}){ }^{78-80}$ Environmental factors such as use of antibiotics, intestinal neurotransmitters/neuromodulators, infectious agents sensory vagal fibers, essential metabolites and pro-inflammatory agents, all convey information to the central nervous system about the intestinal state. The intestinal functions are regulated by gut microbiota to provide health as well as to influence the immune and nervous systems involving brain inflammation via the bidirectional pathways. ${ }^{78}$ The bidirectional communication between the microbiota and the CNS occurs through autonomic, neuroendocrine, enteric, and immune system pathways. ${ }^{79}$

In the microbiota gut-brain- axis, there is interaction and involvement of various afferent or efferent pathways. The hypothalamic-pituitary-adrenal axis, pro-opio-melanotropic neurons (POMC), arcuate nucleus, the central nervous system regulatory areas of satiety, and neuropeptides released from sensory nerve fibers affect the gut microbiota composition directly or through nutrient availability. ${ }^{78}$ These interactions may influence the pathogenesis of all the CMDs and other chronic diseases in which inflammation is implicated. It is possible that an understanding of the relationship between the microbiota gut-brain axis and the neuro-immune systems could be a new approach for understanding the physiopathology and management of chronic noncommunicable disorders. The neurobiological mechanisms through which disruptions in this network may impact health and disease include hypothalamicpituitary-adrenal (HPA)-axis activation, and altered activity of neurotransmitter and immune systems causing oxidative stress and inflammation.

A recent review reported interesting role of nutrients in the modulation of microbiome, brain and the intestinal, epithelium-enteric nervous, endocrine and immune systems. ${ }^{80}$ The most important basis of the gut-brain axis includes; the neuroendocrine-hypothalamicpituitary-adrenal (HPA) axis, neuroanatomy represented by the vagal and spinal afferent neurons, via the gut hormones, immune systems via the multiple pro-inflammatory cytokines, microbially-derived neurotransmitters. The intestinal and brain barriers could be the gate keepers. A failure in the interactions and mutual adaptations predispose a number of autoimmune, neurodegenerative, metabolic, pro-inflamnmatory, mood, behavioral, stress, cognitive, autismspectrum and pain-connected disorders. A prior knowledge of this intricate field, might high light on novel preventive and treatment methods to prevent these disorders. Nutrient administration, alterations in microbiome, reinforcement of enteric and brain barrier and sensing and trafficking modulation may cause improvement leading to promotion in total health [80].Preventive strategies made early in life, such as Mediterranean diets, use of fecal microbiota transplantation, use of psychobiotics and antioxidant flavonoids may be protective for gut microbiota and prevention of CMDs. A high w-6/w-3 ratio in the diet in conjunction with flavonoids as well as probiotics in the diet in a melieu of higher BDNF levels in the gut and the brain could be important in healthy gut-brain-heart-axis for health promotion. ${ }^{81-85}$ A more recent experimental studies has also confirmed the existence of gut-brain and heart axis by demonstrating that increase in inflammation after acute myocardial infarction predicts remodeling and neuro-inflammation. ${ }^{86}$

In brief, gut-brain-axis has already been demonstrated to explain the relation of gut dysfunction with neuropsychiatric dysfunction. The effect of gut dysfunction due to red meat, egg choline by producing gut flora metabolites; TMAO has also been observed indicating the association of gut dysfunction with CMDs. These findings pose the possibility that a brain-gut-heart-axis also exist which also acts bidirectionally; from gut to heart and brain and from brain to heart and gut (inflammation in the beta cells of pancreas) in the development of CMDs. This hypothesis opens a new vista for prevention of CMDs by providing Mediterranean type diets with probiotics which are known to modulate brain function as well as gut function and cardiovascular function.

\section{Acknowledgements}

Logistic support has been provided by the International College of Cardiology for writing this article.

\section{Conflict of interest}

The author declares that there is no conflict of interest.

\section{References}

1. Wang PY, Caspi L, Lam CK, et al. Upper intestinal lipids trigger a gut-brain-liver axis to regulate glucose production. Nature. 2008;452(7190):1012-1016.

2. Singh RB, Chaudhury J, De Meester F, et al. The gut-brain-axis and heart connection. World Heart J. 2010;3:151-174.

3. Singh RB, De Meester F, Wilczynska A, et al. The liver-pancreas and the brain connection in the pathogenesis of obesity and metabolic syndrome. World Herat J. 2010;2(4):319-326.

4. Thaler JP, Cumming DE. Metabolism: food alert. Nature. 2008;452(7190):941-942.

5. Jenkins TA, Nguyen JCD, Polglaze KE, et al. Influence of tryptophan and serotonin on mood and cognition with a possible role of the Gut-Brain Axis. Nutrients. 2016;8(1):pii:E56.

6. Schmidt C. Mental health: thinking from the gut. Nature. 2015;518(7540):S12-15.

7. Smith PA. The tantalizing links between gut microbes and the brain. Nature. 2015;526(7573):312-314. 
8. Wang HX, Wang YP. Gut microbiota-brain axis. Chin Med J (Engl) 2016;129(19):2373-2380.

9. Fedacho J, Singh RB, Pella D, et al. Can gut flora metabolites predict cardiac events and cardiometabolic diseases? World Heart J. 2017;9(2):107-113.

10. Li XS, Obeid S, Klingenberg $\mathrm{R}$, et al. Gut microbiota-dependent trimethylamine N-oxide in acute coronary syndromes: A prognostic marker for incident cardiovascular events beyond traditional risk factors. Eur Heart J. 2017;38(11):814-824.

11. Suzuki T, Heaney LM, Jones DJ, et al. Trimethylamine N-oxide and risk stratification after acute myocardial infarction. Clin Chem. 2017;63(1):420-428.

12. Wang Z, Roberts AB, Buffa JA, et al. Non-lethal inhibition of gut microbial trimethylamine production for the treatment of atherosclerosis. Cell. 2015;163(7):1585-1595.

13. Singh RB, Cornelissen G, shastun S, et al. Atherosclerosis? A disease of the brain. World Heart J. 2017;9:99-106.

14. Shaibani AA, Elkilany G, Singh RB. Stress, peace and the heart. World Heart J. 2015;7(4):265-274.

15. Nabi H, Kivimäki M, Batty GD, et al. Increased risk of coronary heart disease among individuals reporting adverse impact of stress on their health: the Whitehall II prospective cohort study. Eur Heart J. 2013;34 (34):2697-2705.

16. Rosengren A, Hawken S, Ounpuu S, et al. Association of psychosocial risk factors with risk of acute myocardial infarction in 11119 cases, 13648 controls from 52 countries(the INTERHEART Study): case control study. Lancet. 2004;364(9438):953-962.

17. Xu T, Li W, Teo K, et al. Association of psychological risk factors and acute myocardial infarction in China: the INTERHEART China study. Chin Med J (Engl). 2011;124(14):2083-2088.

18. Tawakol A, Ishai A, Takx RA, et al. Relation between resting amygdalar activity and cardiovascular events: a longitudinal and cohort study. Lancet. 2017;389(10071):834-845.

19. Chauveau F, Lange MD, Jüngling K, et al. Prevention of stress-impaired fear extinction through neuropeptide,s action in the lateral amygdala. Neuropsycho-pharmacology. 2012;37(7):1588-1599.

20. Troseid M. Gutmicrobiota and acute coronary syndromes:ready for use in the emergency room? Eur Heart J. 2017;38(11):825-827.

21. Farr S, Baker C, Naples M, et al. Central nervous system regulation of intestinal lipoprotein metabolism by glucagon-like peptide-1 via a BrainGut Axis. Arterioscler Thromb Vasc Biol. 2015;35(5):1092-1100.

22. Farr S, Taher J, Adeli K. Central nervous system regulation of intestinal lipid and lipoprotein metabolism. Curr Opin Lipidol. 2016;27(1):1-7.

23. Taher J, Farr S, Adeli K. Central nervous system regulation of hepatic lipid and lipoprotein metabolism. Curr Opin Lipidol. 2017;28(1):32-38.

24. Wang S, Huang XF, Zhang P, et al. Dietary tea saponin ameliorates alteration of gut microbiota and cognitive decline in diet-induced obese mice. Sci Rep. 2017;27(1):12203.

25. Pedersen HK, Gudmundsdottir V, Nielsen HB, et al. Human gut microbes impact host serum metabolome and insulin sensitivity. Nature. 2016;535(7612):376-381.

26. Wang Z, Klipfell E, Bennett BJ, et al. Gut flora metabolism of phosphatidylcholinepromotes cardiovascular disease. Nature. 2011;472:57-63.
27. Koeth RA, Wang Z, Levison BS, et al. Intestinalmicrobiota metabolism of 1-carnitine, a nutrient in red meat, promotes atherosclerosis. Nat Med. 2013;19(5):576-585.

28. Tang WH, Wang Z, Levison BS, et al. Intestinal microbial metabolism of phosphatidylcholine and cardiovascular risk. $N$ Engl $J$ Med. 2013;368(17):1575-1584.

29. Troseid M, Ueland T, Hov JR, et al. Microbiota-dependent metabolite trimethylamine-N-oxide is associated with disease severity and survival of patients with chronic heart failure. J Intern Med. 2015;277(6):717726.

30. Xiao C, Dash S, Morgantini C, et al. New and emerging regulators of intestinal lipoprotein secretion. Atherosclerosis. 2014; 233(2):608-615.

31. Bercik P, Verdu EF, Foster JA, et al. Chronic gastrointestinal inflammation induces anxiety-like behavior and alters central nervous system biochemistry in mice. Gastroenterology. 2010;139(6):2102-2112.

32. Loftis JM, Huckans M, Morasco BJ. Neuroimmune mechanisms of cytokine-induced depression: current theories and novel treatment strategies. Neurobiol Dis. 2010;37(3):519-533.

33. Graff LA, Walker JR, Bernstein CN. Depression and anxiety in inflammatory bowel disease: a review of comorbidity and management. Inflamm Bowel Dis. 2009;15(7):1105-1118.

34. Maes M. Inflammatory and oxidative and nitrosative stress pathways underpinning chronic fatigue, somatization and psychosomatic symptoms. Curr Opin Psychiatry. 2009;22(1):75-83.

35. O’Mahony L, McCarthy J, Kelly P, et al. Lactobacillus and bifidobacterium in irritable bowel syndrome: symptom responses and relationship to cytokine profiles. Gastroenterology. 2005;128(3):541-551.

36. Cornelissen G. When you eat matters: 60 years of Franz Halberg's nutrition chronomics. The Open Nutra J. 2012;5:16-44.

37. Singh RB, Singh AK, Sharma JP, et al. Nutrition in Chronocardiology: We are Indebted Professor Franz Halberg. The Open Nutr J. 2012;5:4565.

38. Venters HD, Tang Q, Liu Q, et al. A new mechanism of neurodegeneration: pro-inflammatory cytokines inhibits receptor signaling by a survival peptide. Proc Natl Acad Sci U S A. 1999;96(17):9879-9884.

39. Lam CK, Chari M, Su BB, et al. Activation of N-methyl-D-aspartate (NMDA) receptors in the dorsal vagal complex lowers glucose production. J Biol Chem. 2010;285(29):21913-21921.

40. Lauritzen I, Blondeau N, Heurteaux C. Polyunsaturated fatty acids are potent neuroprotectors. EMBO J. 2000;19(8):1784-1788.

41. Singh RB, Pella D, Mechirova V, et al. Can brain dysfunction may be a predisposing factor for metabolic syndrome. Biomed Pharmacother. 2004;58:52-68.

42. Crawford MA, Bazinet RP, Sinclair AJ. Fat intake and CNS functioning: ageing and disease. Ann Nutr Meta. 2009;55(1-3):202-228.

43. Singh RB, Kartik C, Otsuka K, et al. Brain-heart connection and the risk of heart attack. Biomed Pharmacother. 2004;56:257s-265s.

44. O’Donoghue M, Morrow DA, Sabatine MS, et al. Lipoproteinassociated phospholipase A2 and its association with cardiovascular outcomes in patients with acute coronary syndromes in the PROVE IT-TIMI 22 (PRavastatinOratorVastatin Evaluation and Infection Therapy-Thrombolysis In Myocardial Infarction) trial. Circulation. 2006;113(14):1745-1752.

45. Esposito K, Glugliano D. Diet and inflammation:a link to metabolic and cardiovascular diseases. Euro Heart J. 2006;27(1):15-20. 
46. Vogel RA. Eating vascular biology and atherosclerosis: a lot to chew on. Euro Heart J. 2006;27:13-14.

47. Gramenzi A, Gentile A, Fasoli M, et al. Association between certain foods and risk of acute myocardial infarction in women. BMJ. 1990;300(6727):771-773.

48. Singh RB, Pella D, Sharma JP, et al. Increased concentrations of lipoprotein(a), circadian rhythms and metabolic reactions, evoked by acute myocardial infarctions, in relation to large breakfasts. Biomed Pharmacother. 2004;58(Suppl 1):S116-122.

49. Singh RB, Pella D, Neki NS, et al. Mechanism of acute myocardial infarction study. Biomed Pharmacother. 2004;58(Suppl):S111-115.

50. Fung TT, Malik V, Rexroad KM, et al. Sweetened beverage consumption and risk of coronary heart in women. Am J Clin Nutr. 2009; 89(4):10371042.

51. Smith PJ, Blumenthal JA, Babyak MA, et al. Association between n-3 fatty acid consumption and ventricular ectopy after myocardial infarction. Am J Clin Nutr. 2009;89(5):1315-1320.

52. Campos H, Baylin A, Willett WC. Alpha-Linolenic acid and risk of nonfatal acute myocardial infarction. Circulation. 2008;118(4):339-345.

53. Harris WS, Reid KJ, Sands SA, et al. Blood omega-3 and trans fatty acids in middle aged acute coronary syndrome patients. Am J Cardiol. 2007;99(2):154-158

54. Hristova K, Pella D, Singh RB, et al. Sofia declaration for prevention of cardiovascular diseases and type 2 diabetes mellitus: a scientific statement of the international college of cardiology and internationa college of nutrition; ICC-ICN Expert Group. World Heart J. 2014; 6:89106

55. de Lorgeril M, Renaud S, Mamelle N, et al. Mediterranean alpha-linolenic acid-rich diet in secondary prevention of coronary heart disease. Lancet. 1994;343(8911):1454-1459.

56. Renaud S, de Lorgeril M, Delaye J, et al. Cretan Mediterranean die for prevention of coronary heart disease. Am J Clin Nutr. 1995;61(6 Suppl):1360S-1367S.

57. Singh RB, Dubnov G, Niaz MA, et al. Effect of an Indo-Mediterranean diet on progression of coronary disease in high risk patients:a randomized single blind trial. Lancet. 2002;360(9344):1455-1461.

58. Esposito K, Marfella R, Ciotola M, et al. Effect of a Mediterranean-style diet on endothelial dysfunction and markers of vascular inflammation in the metabolic syndrome: a randomized trial. JAMA. 2004;292(12):14401446 .

59. Singh RB, Niaz MA, Agarwal P, et al. Effect of antioxitant rich foods on plasma ascorbic acid, cardiac enzyme and lipid peroxide levels in patients hospitalized with acute myocardial infarction. J Am Diet Assoc. 1995;95(7):775-780.

60. Singh RB, Rastogi SS, Verma R, et al. An Indian experiment with nutritional modulation in acute myocardial infarction. Am $\mathrm{J}$ Cardiol. 1992;69(9):879-885.

61. Singh RB, Rastogi SS, Verma R, et al. Randomized, controlled trial of cardioprotective diet in patients with acute myocardial infarction : results of one year follow up. BMJ. 1992;304(6833):1015-1019.

62. Metzinser D, Digen L, Drewe J, et al. The role of long chain fatty acids in regulating food intake and cholecystokinin release in humans. Gut. 2000;46(5):688-693.

63. Bado A, Levasseur S, Attoub S, et al. The stomach is a source of leptin. Nature. 1998;394(6695):790-793.
64. Barrachina MD, Martinez V, Wang L, et al. Synergistic interaction between leptin and cholecystokinin to reduce short-term food intake in mice. Proc Natl Acad Sci U S A. 1997;94(19):10455-10460.

65. Hirano H, day J, Fibiger HC. Serotonergic regulation of acetylcholine release in rat frontal cortex. J Neurochem. 1995;65(3):1139-1145.

66. Zhou FM, Liang Y, Dani JA. Endogenous nicotinic cholinergic activity regulates dopamine release in the striatum. Nat Neurosci. 2001;4(12):1224-1229.

67. Bartness TJ, Kay Song C, Shi H, et al. Brain-adipose tissue cross talk. Proc Nutr Soc. 2005;64(1):53-64.

68. Huang LZ, Winzer-Serhan UH. Nicotine regulates mRNA expression of feeding peptides in the arcuate nucleus in neonatal rat pups. Dev Neurobiol. 2007;67(3):363-377.

69. Obici S, Feng Z, Morgan M, et al. Central administration of oleic acid inhibits glucose production and food intake. Diabetes. 2002;51(2):271275 .

70. Obici S, Feng Z, Arduini A, et al. Inhibition of hypothalamic carnitine palmitoyltransferase-1 decreases food intake and glucose production. Nat Med. 2003;9(6):756-761.

71. Aron-Wisnewsky J, Clément K. The gut microbiome, diet, and links to cardiometabolic and chronic disorders. Nat Rev Nephrol. 2016; 12(3):169-181

72. Org E, Blum Y, Kasela S, et al. Relationships between gut microbiota, plasma metabolites, and metabolic syndrome traits in the METSIM cohort. Genome Biology. 2017;18(1):70.

73. Senthong $\mathrm{V}$, Wang $\mathrm{Z}$, Li XS, et al. Intestinal microbiota-generated metabolite trimethylamine-N-oxide and 5-year mortality risk in stable coronary artery disease: The Contributory Role of Intestinal Microbiota in a COURAGE-Like Patient Cohort. J Am Heart Assoc. 2016; 5(6).

74. Tang WHW, Wang Z, Kennedy DJ, et al. Gut Microbiota-Dependent Trimethylamine $N$-oxide (TMAO) Pathway Contributes to Both Development of Renal Insufficiency and Mortality Risk in Chronic Kidney Disease. Circ Res. 2015;116(3):448-455.

75. MenteA, ChalcraftK, AkH, etal. Therelationshipbetween trimethylamine$\mathrm{N}$-oxide and prevalent cardiovascular disease in a multiethnic population living in Canada. Can J Cardiol. 2015;31(9):1189-1194.

76. Warrier M, Shih DM, Burrows AC, et al. The TMAO-generating enzyme flavin monooxygenase 3 is a central regulator of cholesterol balance. Cell Rep. 2015;10:326-338.

77. Clapp M, aurora N, Herrera L, et al. Gut microbiota's effect on mental health: The gut-brain axis. Clin Pract. 2017;7(4):987.

78. Petra AI, Panagiotidou S, Hatziagelaki E, et al. Gut-microbiota-brain axis and its effect on neuropsychiatric disorders with suspected immune dysregulation. Clin Ther. 2015;37(5):984-995.

79. Rieder R, Wisniewski PJ, Alderman BL, et al. Microbes and mental health: A review. Brain Behav Immun. 2017;66:9-17.

80. Lerner A, Neidhöfer S, Matthias T. The Gut Microbiome Feelings of the Brain: A Perspective for Non-Microbiologists. Microorganisms. $2017 ; 5(4)$.

81. Toda E, Toru T, Singh RB, et al. Can low w-6/w-3 ratio Paleolithic style diet stop cardiovascular diseases? Tissue is the Issue. Amer Med J. 2012;3:183-193.

82. Cai D, Holm JM, Duignan IJ, et al. BDNF-mediated enhancement of inflammation and injury in the aging heart. Physiol Genomics. 2006;24(3):191-197. 
83. Rao JS, Ertley RN, Lee HJ, et al. n-3 polyunsaturated fatty acid deprivation in rats decreases frontal cortex BDNF via a p38 MAPKdependent mechanism .Mol Psychiatry 2007;12(1):36-46.

84. Bischoff SC. Gut Health: a new objective in medicine? BMC Med. 2011;9:24
85. Preidis GA, Versalovic J. Targeting the human microbiome with antibiotics, probiotics, and prebiotics: gastroenterology enters the metagenomics era. Gastroenterology. 2009;136(6):2015-2031.

86. Thackeray JT, Hupe HC, Wang Y, et al. Myocardial Inflammation after acute myocardial infarction predicts remodeling and neuro-inflammation. JACC. 2018;71(3):263-275. 